\title{
Clinical Comparative Study: Efficacy and Tolerability of Tolperisone and Thiocolchicoside in Acute Low Back Pain and Spinal Muscle Spasticity
}

\author{
Rajeev Rao ${ }^{1}$, Atul Panghate ${ }^{2}$, Ajay Chandanwale ${ }^{3}$, Indrajeet Sardar ${ }^{4}$, Mriganka Ghosh $^{5}$, Modan Roy $^{6}$, \\ Bireswar Banerjee ${ }^{5}$, Ankur Goswami ${ }^{7}$, Prakash P. Kotwal ${ }^{7}$ \\ ${ }^{1}$ Department of Orthopedics, Jubilee Missions Medical College, Thrissur, India \\ ${ }^{2}$ Department of Orthopedics, Sanjeevani Hospital, Mumbai, India \\ ${ }^{3}$ Department of Orthopedics, Sir JJ Group of Hospitals, Mumbai, India \\ ${ }^{4}$ Department of Orthopedics, Nightingale Hospital, Kolkata, India \\ ${ }^{5}$ Department of Orthopedics, Kothari Medical Center, Kolkata, India \\ ${ }^{6}$ Department of Orthopaedics, Kolkata Medical College, Kolkata, India \\ ${ }^{7}$ Department of Orthopaedics, All India Institute of Medical Sciences (AIIMS), Delhi, India
}

Study Design: We performed a multicentric, randomized,comparative clinical trial. Eligible patients were randomly assigned to receive $150 \mathrm{mg}$ of Tolperisone thrice daily or $8 \mathrm{mg}$ of Thiocolchicoside twice daily for 7 days.

Purpose: To assess the efficacy and tolerability of Tolperisone in comparison with Thiocolchicoside in the treatment of acute low back pain with spasm of spinal muscles.

Overview of Literature: No head on clinical trial of Tolperisone with Thiocolchicoside is available and so this study is done.

Methods: The assessment of muscle spasm was made by measuring the finger-to-floor distance (FFD),articular excursion in degrees on performing Lasegues maneuver and modified Schobers test. Assessment of pain on movement and spontaneous pain (pain at rest) of the lumbar spine was made with the help of visual analogue scale score.

Results: The improvement in articular excursion on Lasegues maneuver was significantly greater on day $3(p=0.017)$ and day $7(p=0.0001)$ with Tolperisone as compared to Thiocolchicoside. The reduction in FFD score was greater on day 7 $(p=0.0001)$ with Tolperisone. However there was no significant difference in improvement in Schobers test score on day 3 $(p=0.664)$ and day $7(p=0.192)$. The improvement in pain score at rest and on movement was significantly greater with Tolperisone $(p=0.0001)$.

Conclusions: Tolperisone is an effective and well tolerated option for treatment of patients with skeletal muscle spasm associated with pain.

Key Words: Tolperisone, Thiocolchicoside, Skeletal muscle relaxant, Low back pain, Muscle, Spasm

\section{Introduction}

Generally, the symptoms of most of the uncomplicated low back pain (LBP) are managed with short term use of non-steroidal inflammatory drugs (NSAIDs) and centrally acting skeletal muscle relaxants [1]. Unfortunately NSAIDs have unfavorable gastric tolerability profile whereas most of the centrally acting muscle relaxants have central nervous system side effects such as sedation, dizziness, impairment of coordination, mental confusion, weakness, withdrawal

Received Aug 10, 2011 Revised Oct 18, 2011 Accepted Oct 19, 2011

Corresponding author: Prakash P. Kotwal, MS, FAMS, FIMSA

Department of Orthopedics, All India Institute of Medical Science (AIIMS),

Ansari Nagar, New Delhi, Delhi-110029, India

Tel: +91 9868129599, Fax: +91 011 2698663, E-mail: prakash.kotwal@gmail.com

Copyright (C) 2012 by Korean Society of Spine Surgery

This is an Open Access article distributed under the terms of the Creative Commons Attribution Non-Commercial License (http://creativecommons.org/licenses/by-nc/3.0/) which permits unrestricted non-commercial use, distribution, and reproduction in any medium, provided the original work is properly cited. Asian Spine Journal • pISSN 1976-1902 eISSN 1976-7846 
phenomenon or anti-cholinergic adverse effects which results in non-compliance to treatment and affects ability to work [2].

The evidence of spinal muscle spasm associated acute back pain justifies the use of centrally acting muscle relaxant in treatment of acute low back pain. Tolperisone hydrochloride, a piperidine derivative, is centrally acting muscle relaxant and has been in clinical use for decades in Europe and Asia [3]. It acts by inhibiting the pathologic mono- and polysynaptic reflex activity in the formation reticularis and spinal cord and by stabilizing the nerve membrane [4] in a dose dependant manner [2]. It differs from other myotonolytic agents due to its pharmacological properties which mediate muscle relaxation without concomitant sedation or withdrawal phenomenon [3-5]. Unlike other centrally acting skeletal muscle relaxants, it has no substantial affinity to adrenergic, cholinergic, dopaminergic or serotonergic receptors in the central nervous system.

Moreover Tolperisone has analgesic activity in humans, which could be attributed to its chemical structure, the tertiary aryl amine producing a Lidocaine like activity of stabilizing nerve membranes [2]. Use of central muscle relaxants with long duration of action have the risk of neuromuscular blockade, hence muscle relaxants with short and intermediate duration of action like Tolperisone should be preferred [1].

In several clinical studies Tolperisone has been shown to relieve muscle spasms associated with diseases of the spinal column or proximal joints [1,6-10]. However, no comparative data on Tolperisone with other centrally acting muscle relaxants is available. Thiocolchicoside (TC), a natural glycoside is a centrally acting skeletal muscle relaxant used in the management of low back pain. TC is slightly more effective and scored significantly better than Tizanidine (TZ) [11] or is at least as effective as TZ in the treatment of acute LBP [12]. Hence TC being a commonly used skeletal muscle relaxant was used as the comparator in this study. This study was conducted with the objective to assess efficacy and tolerability of Tolperisone in comparison with $\mathrm{TC}$ in treatment of acute low back pain with spasm of spinal muscles.

\section{Materials and Methods}

\section{Study design}

This was a prospective, multi-centric, randomized, open label comparative clinical trial conducted at JJ group of Hospitals, Mumbai; AIIMS, New Delhi; Sanjeevani Hospital, Mumbai; Jubilee Missions Medical College, Thrissur; and 3 centers in Kolkata; Kolkata Medical College, Kothari Medical Center and Nightingale Hospital. The study protocol was approved by the institutional review board at each centre, and all patients provided written informed consent. The study was conducted according to the International Conference on Harmonisation of Technical Requirements for Registration of Pharmaceuticals for Human Use-Good Clinical Practice Guidelines with strict adherence to the protocol.

\section{Subject selection criteria}

Patients attending the outpatient department were screened and assessed according to the specified inclusion and exclusion criteria. A total of 250 eligible patients, of either sex between 18 to 45 years of age, with spasm of spinal muscles with acute or relapsing low back pain, of moderate to severe intensity and no finding of severe spinal diseases, willing to take medications as directed and come for the follow-up were included in the study.

Patients having other lumbar spinal tract conditions such as spondylitis, fracture, cancer, severe arthritis and osteoporosis; muscular diseases such as myositis, poliomyositis, muscular dystrophia and myotonia; and other severe diseases affecting the neurological or cardiovascular systems, liver and kidneys were not enrolled. Patients who had taken any form of skeletal muscle relaxant in previous 7 days and those with hypersensitivity to any of the ingredients of the test formulations were not recruited in the study. Pregnant or lactating women or women of child bearing potential not following adequate contraceptive measures were excluded.

\section{Treatment procedure}

Eligible patients were randomized to receive either Tolperisone hydrochloride $150 \mathrm{mg}$ thrice daily or TC $8 \mathrm{mg}$ twice daily until the patient got relief of muscle spasm and pain but not more than 7 days.

During the trial antacids, $\mathrm{H}_{2}$ blockers or proton pump inhibitors were prescribed. Concomitant treatment in the form of diclofenac sodium was given to or taken by the patient was recorded in the case report form. No other medicines were allowed. 
Table 1. Demography and baseline characteristics of patients at baseline

\begin{tabular}{lcc}
\hline \hline \multicolumn{1}{c}{ Parameter } & Tolperisone group & Thiocolchicoside group \\
\hline Mean age (yr) & $35.67 \pm 7.82$ & $34.68 \pm 7.81$ \\
Mean weight (kg) & $67.24 \pm 11.33$ & $65.54 \pm 9.45$ \\
Mean height (cm) & $164.21 \pm 10.15$ & $164.91 \pm 9.66$ \\
Pulse rate (beats/min) & $78.25 \pm 7.04$ & $78.03 \pm 7.47$ \\
Systolic blood pressure (mm Hg) & $118.91 \pm 10.83$ & $120.38 \pm 10.82$ \\
Diastolic blood pressure (mm Hg) & $78.32 \pm 7.09$ & $80.17 \pm 12.20$ \\
\hline
\end{tabular}

Values are presented as mean \pm standard deviation.

\section{Efficacy assessments}

Assessment of muscle spasm was carried out on at day 1 (day of screening and enrolment: visit 1), day 3 (visit 2) and day 7 (visit 3) by following procedures: 1) Finger-tofloor distance (FFD): It was measured by flexion at hip joint in standing position. The patient was made to bend down as far as possible without bending the knees. The distance between the floor and fingertips was measured. The reduction in the FFD (in cm) on relief of muscle spasm (post drug treatment) was measured. 2) Lasegue's manoeuvre: In this test articular excursion of the hip in degrees on performing Lasegue's manoeuvre before inducing pain in supine position which involved gradually raising of lower extremity by flexing the hip with the knee in extension passively. The Laseague's manoeuvre measured the angle between the raised limb and tabletop. The increase in degree of articular excursion on relief of spasm (post treatment) was measured. 3) Modified Schober's test: The examiner stood behind the subject and made three marks; one mark behind lumbosacral junction; a second mark on a spinous process $10 \mathrm{~cm}$ above first mark (measure to the nearest millimetre) and a third mark $5 \mathrm{~cm}$ below the first mark. The distance between inferior and superior most marks was measured, with " 0 " at inferior mark. Pressing the measuring tape against subject's lower back, having them bend forward keeping the knees straight, and the distance between most superior and inferior marks at the end of the range of movement (ROM) was recorded. The ROM to be recorded as the assessment was the difference between the $15 \mathrm{~cm}$ and the length measured at the end of the motion.

Assessment of spontaneous pain intensity and pain on movement was carried out on day 1 (visit 1), day 3 (visit 2) and day 7 (visit 3) by means of a $10 \mathrm{~cm}$ visual analogue scale (VAS) as reported by a patient between 0 (no pain) to 10 (unbearable pain).
Global Assessment of efficacy of treatment was done at the end of the study by investigator and of tolerability by patients by means of a 4-point scale (excellent, good, satisfactory and poor).

\section{Statistical analysis}

A sample size of 250 completed patients was needed (125 per treatment group) at a power of $90 \%$ and level of significance of $95 \%(\alpha=0.05)$ to detect a difference of $>20 \%$ for reduction in FFD distance from baseline on day 5 between Tolperisone and TC assuming that Tolperisone achieves a reduction of $>20 \%$ compared to TC. Results are expressed as mean and standard deviation (SD) for continuous data, and numbers \& percentages for discrete data. Comparison of two groups was done by Student's $t$-test for parametric data (FFD, VAS score, articular excursion). Mann-Whitney $U$-test for non-parametric data (rescue drug, global assessment). Pearson's chi-square test was used for overall assessment of efficacy and tolerability.

\section{Results}

\section{Demographics}

A total of 289 patients were screened, of which, 250 were randomized to either Tolperisone $(\mathrm{n}=125)$ therapy or TC ( $n=125)$ and were followed for up to 7 days. Both treatment groups were comparable with respect to demographic and baseline characteristics (Table 1).

The patient compliance for study medication was similar in both the study groups. In Tolperisone group 25 patients required rescue medication whereas 52 patients required rescue medication in TC group. 
Table 2. Response to Tolperisone and Thiocolchicoside on day 3 and day 7 of treatment on finger-to-floor distance, Laseague's Maneuver score and Modified Schober's test score

\begin{tabular}{lccc}
\hline \multicolumn{1}{c}{ Parameter } & $\begin{array}{c}\text { Tolperisone group } \\
(\mathrm{n}=125)\end{array}$ & $\begin{array}{c}\text { Thiocolchicoside group } \\
(\mathrm{n}=125)\end{array}$ & $p$-value \\
& & & \\
Finger-to-floor distance $(\mathrm{cm})$ & $39.72 \pm 29.20$ & $32.85 \pm 28.28$ & 0.393 \\
Baseline & $26.67 \pm 21.50$ & $16.80 \pm 11.77$ & 0.145 \\
Day 3 & $11.97 \pm 9.83$ & & 0.001 \\
Day 7 & & $43.92 \pm 17.49$ & 0.964 \\
Laseague's maneuver score & $43.82 \pm 16.95$ & $55.53 \pm 15.32$ & 0.017 \\
Baseline & $59.92 \pm 13.51$ & $67.37 \pm 14.57$ & 0.001 \\
Day 3 & $73.29 \pm 9.85$ & & 0.398 \\
Day 7 & & $3.32 \pm 1.33$ & 0.644 \\
Modified Schober's test score (cm) & $3.47 \pm 1.34$ & $4.89 \pm 1.66$ & 0.192 \\
Baseline & $5.00 \pm 1.99$ & $6.47 \pm 2.67$ & \\
Day 3 & $6.95 \pm 3.08$ & & \\
Day 7 & & & \\
\hline
\end{tabular}

Values are presented as mean \pm standard deviation.

Table 3. Effect of Tolperisone and Thiocolchicoside on pain at rest and on movement on day 3 and day 7 post treatment

\begin{tabular}{lccc}
\hline \hline & Tolperisone & Thiocolchicoside & $p$-value \\
\hline Pain at rest & & & \\
Baseline & $6.46 \pm 1.59$ & $6.54 \pm 1.50$ & 0.680 \\
Day 3 & $4.22 \pm 1.51$ & $4.68 \pm 1.52$ & 0.018 \\
Day 7 & $2.19 \pm 1.32$ & $2.98 \pm 1.33$ & 0.0001 \\
Pain on ovement & & \\
Baseline & $7.72 \pm 1.40$ & $7.73 \pm 1.49$ & 0.959 \\
Day 3 & $5.27 \pm 1.69$ & $5.92 \pm 1.50$ & 0.003 \\
Day 7 & $2.99 \pm 1.43$ & $3.94 \pm 1.46$ & 0.0001 \\
\hline
\end{tabular}

Values are presented as mean \pm standard deviation score.

\section{1) Efficacy assessment}

(1) Assessment of muscle spasm

FFD: Both the medications demonstrated a significant effect on the FFD which appeared to be similar. The mean change in FFD from the baseline on day 3 and day 7 in Tolperisone and TC groups respectively was statistically significant. The mean $( \pm \mathrm{SD})$ distance decreased from 39.72 $( \pm 29.20) \mathrm{cm}$ at baseline to $11.97( \pm 9.83) \mathrm{cm}$ in Tolperisone group and from $42.85( \pm 28.28) \mathrm{cm}$ at baseline to 16.80 $( \pm 11.77) \mathrm{cm}$ during the treatment with TC at day 7 (Table 2). On comparison of percentage change of FFD with Tolperisone $(-32.86 \%)$ and TC $(-28.71 \%)$, was not statistically significant on day $3(p=0.145)$, but percentage change of FFD with Tolperisone (-69.87\%) and TC (-60.80\%) was statistically significant on day $7(p=0.001)$ (Fig. 1).

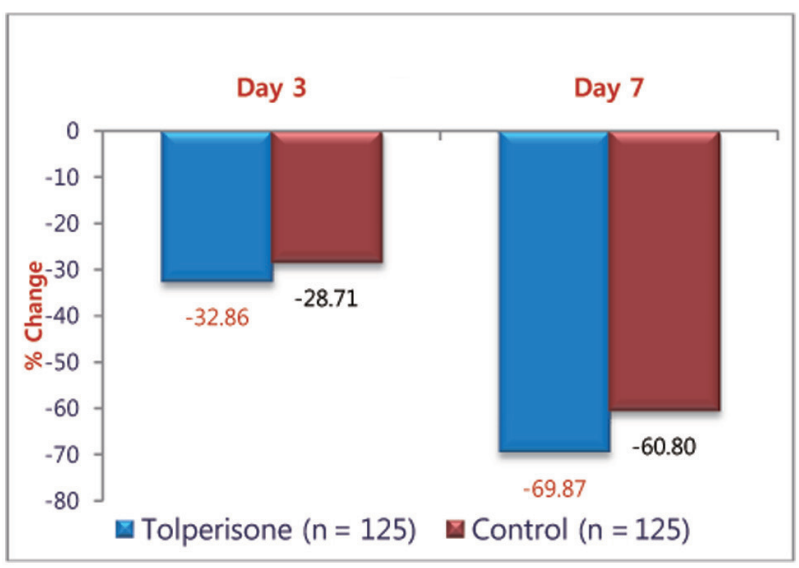

Fig. 1. Percentage change in finger-to-floor distance $(\mathrm{cm})$ with Tolperisone and Thiocolchicoside. 
(2) Laseague's manoeuvre score

The muscle relaxant effect of Tolperisone and TC was demonstrated by Lasegue's manoeuvre score. The mean Lasegue's Manoeuvre score with the treatments, Tolperisone and TC, significantly improved from the baseline on day 3 and day 7 (Table 1). On comparison of percentage improvement in the change of Laseague's Manoeuvre Score with Tolperisone (36.74\%) and TC (26.43\%) on day $3(p=0.017)$ was statistically significant (Fig. 2$)$. It was also was statistically significant on day $7(p=0.001)$, Tolperisone $(67.24 \%)$ and TC (53.38\%).

\section{(3) Modified Schober's test score}

The muscle relaxant activity of both the medication was demonstrated as observed from the change in mean Schober's Test Score form baseline on day 3 and day 7. The improvement in Schober's Test Score was greater with Tolperisone but not statistically significant on day 3 ( $p=0.644)$ and day 7 ( $p=0.192)$ (Table 2). Percentage change in mean

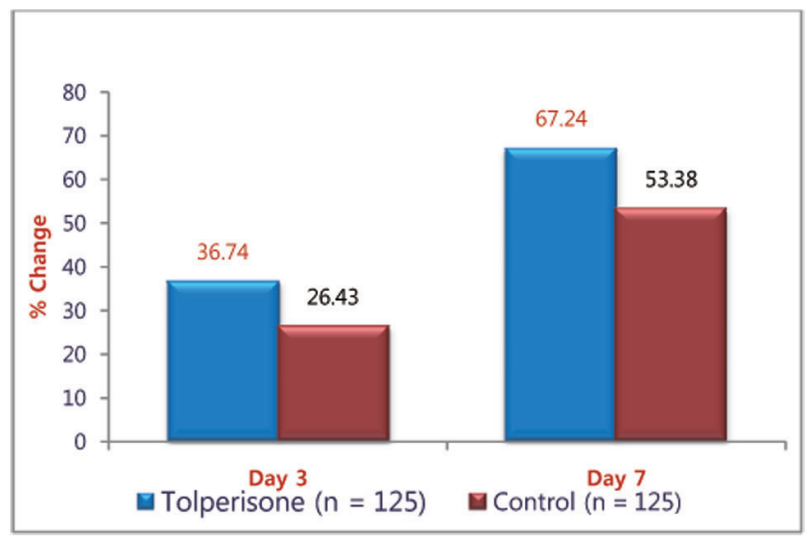

Fig. 2. Change in mean Laseague's manoeuvre score with Tolperisone and Thiocolchicoside.

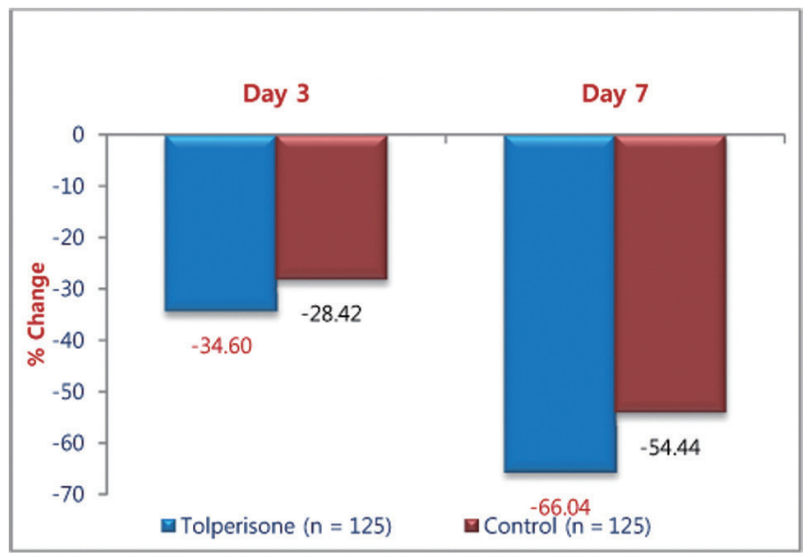

Fig. 4. Percentage change in mean pain at rest with Tolperisone and Thiocolchicoside.
Schober's Test Score on treatment with Tolperisone and TC was observed in both groups is depicted in Fig. 3 .

\section{(4) Pain at rest score}

The effects of the both medications on spontaneous pain are presented in Table 2 . The mean $( \pm \mathrm{SD})$ VAS score decreased from $6.46( \pm 1.59)$ at the baseline evaluation to 2.19 $( \pm 1.32)$ at the end of the treatment in patients receiving Tolperisone. The improvement in pain at rest score was greater with Tolperisone which was significant on day $3(p=0.018)$ and day 7 ( $p=0.0001)$ (Table 3$)$. Percentage change in mean score for pain at rest with Tolperisone and TC is represented in Fig. 4.

\section{(5) Pain on movement score}

The mean $( \pm \mathrm{SD})$ pain on movement score improved from $7.72( \pm 1.40)$ at baseline to $2.99( \pm 1.43)$ in Tolperisone group and $7.73( \pm 1.49)$ at baseline to $3.94( \pm 1.46)$ in TC group at day 7 . The improvement in pain at rest score was

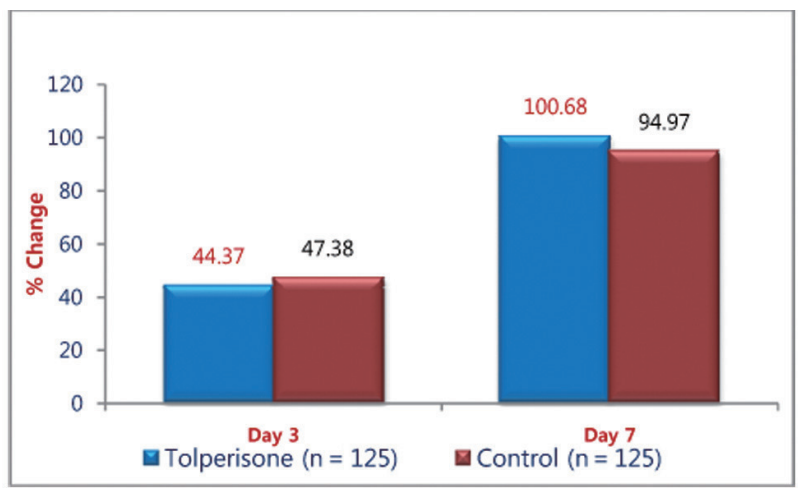

Fig. 3. Percentage change in mean Schober's test score on treatment with Tolperisone and Thiocolchicoside.

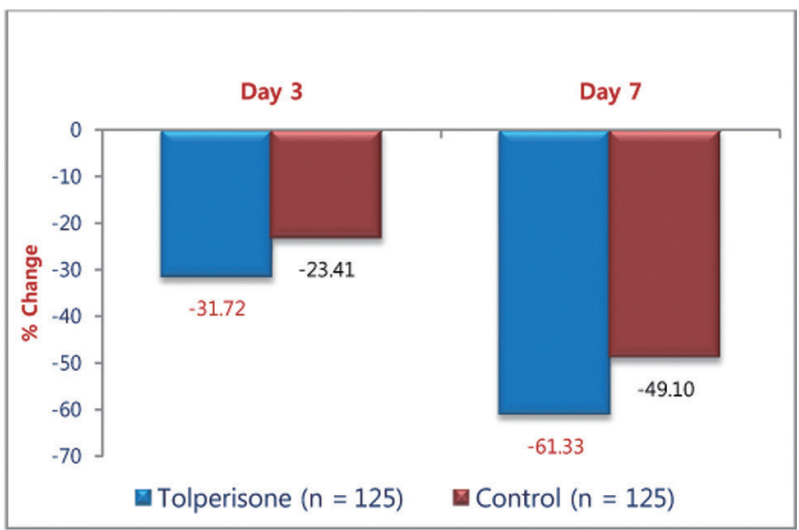

Fig. 5. Percentage change in mean pain score on movement with Tolperisone and Thiocolchicoside. 
greater with Tolperisone on day $3(p=0.003)$ and day 7 $(p=0.0001)$ which was statistically significant (Table 3$)$. Percentage change in mean pain score at rest movement with Tolperisone and TC is shown in Fig. 5.

\section{(6) Rescue medication}

The rescue medication in the form of diclofenac sodium was provided to 16 patients in the Tolperisone group and 39 patients in TC group.

(7) Global efficacy assessment by physicians

As per the assessment by physician Tolperisone produced good to excellent efficacy in $90.25 \%$ of patients where as thiocolchiocoside produced good to excellent efficacy in $73.33 \%$ of patients (Fig. 6).

\section{Safety and tolerability assessments}

The evaluation of the adverse drug reactions occurring during the trial showed a significant tolerability in favour of Tolperisone. As per the global assessment by patients Tolperisone produced good to excellent efficacy in $89.9 \%$ of patients where as thiocolchiocoside produced good to excellent efficacy in $52.5 \%$ of patients (Fig. 7).

Only 7 patients out of 125 suffered from side effects in Tolperisone group compared to 14 patients out of 125 who suffered from side effects in Thiocolchicocide group. The adverse events experienced with Tolperisone were loose motion $(n=1)$, nausea $(n=2)$, giddiness $(n=2)$, abdominal pain $(\mathrm{n}=1)$, itching $(\mathrm{n}=1)$ and with TC were loose motion $(n=8)$, skin rash $(n=1)$ nausea $(n=1)$, giddiness $(n=4)$. All adverse events were of mild intensity and resolved without any intervention. They were reported on day 3 and resolved on day 7 of trial.

\section{Discussion}

Tolperisone has been evaluated and found to be effective for the treatment of several musculoskeletal disorders like, cervicobrachial myofascial pain syndrome, post-stroke spasticity, back pain, multiple sclerosis, vertebrogenic algesic syndromes, neuroleptic syndrome, neurolathyrism, neurosensory hypoacusis, painful reflex muscle spasm, muscular and vascular complaints accompanying climacterics. It is also used as an adjuvant to complex therapy, in 300 to 450 mg daily doses, for the treatment of locomotor diseases accompanied by muscular hypertonia, muscular spasticity, and contracture [2].

Tolperisone, a centrally acting muscle relaxant (CMR) free from side effects, first described by Nador and Porszasz in 1958, is a clinically useful drug for relieving spasticities of neurological origin and muscle spasms associated with painful locomotor diseases. Its first description classified it as an antinicotinic drug based on the fact that it effectively inhibited nicotine induced lethality. Nevertheless, it also inhibited convulsions evoked by electroshock, pentylenetetrazole or strychnine. Its most characteristic effect, however, is potent inhibition of mono and polysynaptic spinal reflexes, and Ono et al. [13] reported that a "membrane stabilizing" effect may underlie its pharmacological actions. Tolperisone acts as an acute blocker of voltage dependent sodium channels.

Several randomized controlled clinical trials have demonstrated that Tolperisone has superior efficacy as compared to placebo in the treatment of spastic hypertonia following cerebral stroke [6-8]. In this indication an individual dose titration which may exceed the recommended maximum dose of $450 \mathrm{mg}$ daily results in optimized therapeutic benefit [6$8]$.

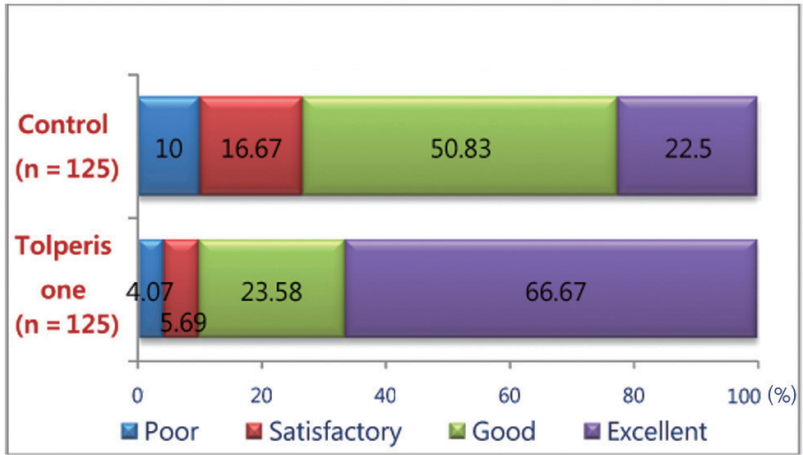

Fig. 6. Global efficacy assessment by physician (\% of patients) with Tolperisone and thiocolchicoside.

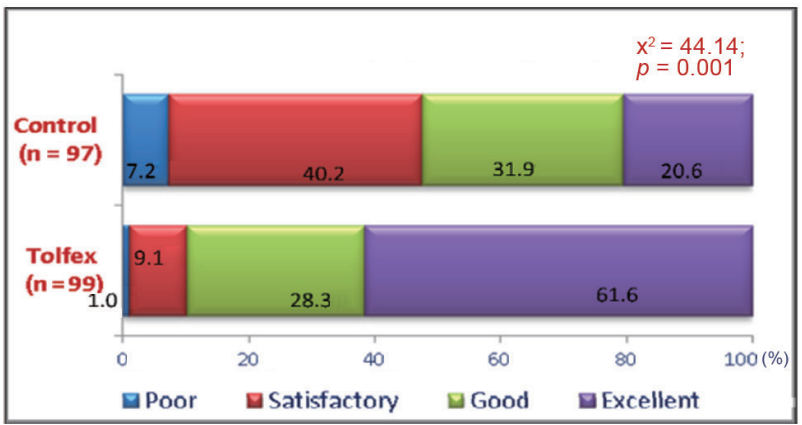

Fig. 7. Global assessment of tolerability by patients (\% of patients) with Tolperisone and thiocolchicoside. 
The skeletal muscle relaxant and analgesic activity of Tolperisone has been evidenced in experimental jaw-muscle pain [9]. When used as prophylactic, Tolperisone reduced isometric force in post-exercise muscle soreness [5].

Adverse events experienced were less with Tolperisone as compared to placebo and were mostly of mild-to-moderate intensity. Tolperisone has a good tolerability and no withdrawals caused by adverse events with Tolperisone [6-9,11]. Single and repeated doses of Tolperisone hydrochloride $150 \mathrm{mg}$ showed that Tolperisone hydrochloride, although being a centrally active muscle relaxant, does not cause any sedation and does not impair reaction times [4].

Skeletal muscle relaxant activity of oral Tolperisone has been evaluated in a prospective, randomized, double-blind, placebo-controlled in the treatment of painful reflex muscle spasm associated with diseases of the spinal column or proximal joints. In this study a total of 138 patients, aged between 20 and 75 years, were enrolled in 8 rehabilitation centers. Patients were randomized to receive either $300 \mathrm{mg}$ Tolperisone hydrochloride or placebo for a period of 21 days. Both treatment groups recovered during the 3 weeks rehabilitation program. However, Tolperisone hydrochloride proved to be significantly superior to placebo treatment. $(p=0.03)$ [2].

$\mathrm{TC}$, a natural glycoside is a centrally acting skeletal muscle relaxant used in the management of low back pain. In clinical trial conducted in Indian population, it was found that Tolperisone was slightly more effective and scored significantly better than TZ [10]. Another study showed that $\mathrm{TC}$ was at least as effective as TZ in the treatment of acute LBP, while it appears devoid of any sedative effect in contrast to TZ [12]. Hence TC being a commonly used skeletal muscle relaxant was used as the comparator in this study.

Our results demonstrate that Tolperisone is an effective muscle relaxant agent with efficacy similar to that of other compounds, such as TC, which are currently used in the management of low back pain due to a spinal muscles spasm. The results to this study showed that Tolperisone $150 \mathrm{mg}$ provides a better clinical response rates and symptom control over TC $8 \mathrm{mg}$ in patients with acute low back pain with spinal muscle spam. This could be explained by the fact that Tolperisone acts by more than one mechanism: inhibiting the pathologic mono- and polysynaptic reflex activity in the formation reticularis and spinal cord and by stabilizing the nerve membrane. Moreover Tolperisone has analgesic activity in humans, which could be attributed to its chemical structure, the tertiary aryl amine producing a
Lidocaine like activity of stabilizing nerve membranes.

The score differences in Laseague's maneuver, pain at rest for both day 3 and day 7 and pain on movement for day 7 , showed significantly better results in Tolperisone group. Other muscle spasm assessment parameters also showed better improvement in Tolperisone; however not statistically significant for both day 3 and day 7. The limitations of the study may be was short follow-up and absence of a control group. The need of rescue medication was significantly greater in TC group than that of in Tolperisone group. Global impression on efficacy by doctors and tolerability by patients show significant differences at the end of the study $(p<0.001)$ the adverse events profile was similar to that documented in earlier studies.

Another consistent advantage of Tolperisone over TC and other muscle relaxant agents is represented by tolerance; in this study, the adverse events were mild to moderate in intensity. On global assessment of tolerability $91.87 \%$ of the patients graded Tolperisone as good to excellent. This could be because unlike other centrally acting skeletal muscle relaxants, it has no substantial affinity to adrenergic, cholinergic, dopaminergic or serotonergic receptors in the central nervous system.

\section{Conclusions}

Tolperisone is an effective skeletal muscle relaxant agent with efficacy similar to TC and relatively better tolerated option for treatment of patients with low back pain associated with muscle spasm.

\section{Acknowledgements}

This study was an Investigator Sponsored Trial. The authors would like to thank Dr. Deepak Langde, for statistical analysis and data management. The clinical trial complies with the current laws of the country in which it was performed.

\section{REFERENCES}

1. Cabitza P, Randelli P. Efficacy and safety of eperisone in patients with low back pain: a double blind randomized study. Eur Rev Med Pharmacol Sci 2008;12:22935.

2. Pratzel HG, Alken RG, Ramm S. Efficacy and tolerance of repeated oral doses of tolperisone hydrochloride 
in the treatment of painful reflex muscle spasm: results of a prospective placebo-controlled double-blind trial. Pain 1996;67:417-25.

3. Quasthoff S, Möckel C, Zieglgänsberger W, Schreibmayer W. Tolperisone: a typical representative of a class of centrally acting muscle relaxants with less sedative side effects. CNS Neurosci Ther 2008;14:10719.

4. Dulin J, Kovács L, Ramm S, Horvath F, Ebeling L, Kohnen R. Evaluation of sedative effects of single and repeated doses of $50 \mathrm{mg}$ and $150 \mathrm{mg}$ tolperisone hydrochloride. Results of a prospective, randomized, doubleblind, placebo-controlled trial. Pharmacopsychiatry 1998;31:137-42.

5. Bajaj P, Arendt-Nielsen L, Madeleine P, Svensson P. Prophylactic tolperisone for post-exercise muscle soreness causes reduced isometric force: a doubleblind randomized crossover control study. Eur J Pain 2003;7:407-18.

6. Koval'chuk VV, Skoromets AA, Vasil'eva IV. Comparative efficacy of different muscle relaxants in the rehabilitation of post-stroke patients with spasticity. $\mathrm{Zh}$ Nevrol Psikhiatr Im S S Korsakova 2008;108:14-9.

7. Stamenova P, Koytchev R, Kuhn K, et al. A randomized, double-blind, placebo-controlled study of the efficacy and safety of tolperisone in spasticity following cerebral stroke. Eur J Neurol 2005;12:453-61.

8. Stamenova P, Koytchev R, Kuhn K, et al. A randomized, double blind, placebo-controlled study of the efficacy and safety of tolperisone in spasticity following cerebral stroke. Zh Nevrol Psikhiatr Im S S Korsakova 2006;106:34-42.

9. Svensson P, Wang K, Arendt-Nielsen L. Effect of muscle relaxants on experimental jaw-muscle pain and jawstretch reflexes: a double-blind and placebo-controlled trial. Eur J Pain 2003;7:449-56.

10. Melka A, Tekle-Haimanot R, Lambien F. Symptomatic treatment of neurolathyrism with tolperisone $\mathrm{HCL}$ (Mydocalm): a randomized double blind and placebo controlled drug trial. Ethiop Med J 1997;35:77-91.

11. Soonawalla DF, Joshi N. Efficacy of thiocolchicoside in Indian patients suffering from low back pain associated with muscle spasm. J Indian Med Assoc 2008;106:3315.

12. Ketenci A, Ozcan E, Karamursel S. Assessment of efficacy and psychomotor performances of thiocolchicoside and tizanidine in patients with acute low back pain. Int J Clin Pract 2005;59:764-70.

13. Ono H, Fukuda H, Kudo Y. Mechanisms of depressant action of muscle relaxants on spinal reflexes: participation of membrane stabilizing action. J Pharmacobiodyn $1984 ; 7: 171-6$ 